\title{
TUTORIAL: Creating Repeatable, Reusable Experimentation Pipelines With Popper
}

\author{
Ivo Jimenez \\ UC Santa Cruz \\ ivo.jimenez@ucsc.edu
}

\author{
Jay Lofstead \\ Sandia National Laboratories \\ gflofst@sandia.gov
}

\author{
Carlos Maltzahn \\ UC Santa Cruz \\ carlosm@ucsc.edu
}

\begin{abstract}
Popper is an experimentation protocol for conducting scientific explorations and writing academic articles following a DevOps approach. The Popper CLI tool helps researchers automate the execution and validation of an experimentation pipeline. In this tutorial we give an introduction to the concepts and CLI tool, and go over hands-on exercises that help.
\end{abstract}

\section{* CCS Concepts• Software and its engineering $\rightarrow$ Soft- ware verification and validation;}

\section{ACM Reference Format:}

Ivo Jimenez, Jay Lofstead, and Carlos Maltzahn. 2019. TUTORIAL: Creating Repeatable, Reusable Experimentation Pipelines With Popper. In PPoPP '19: Symposium on Principles and Practice of Parallel Programming, February 16-20, 2019, Washington, DC, USA. ACM, New York, NY, USA, 2 pages. https://doi.org/10.1145/3293883. 3302575

\section{Introduction}

Currently, approaches to scientific research require activities that take up much time but do not actually advance our scientific understanding. For example, researchers and their students spend countless hours reformatting data and writing code to attempt to reproduce previously published research. What if the scientific community could find a better way to create and publish our workflows, data, and models to minimize the amount of the time spent "reinventing the wheel"? Popper ${ }^{1}$ [1] is a protocol and CLI tool ${ }^{2}$ for implementing scientific exploration pipelines following a DevOps ${ }^{3}$ approach. This allows researchers to generate work that is easy to reproduce.

\footnotetext{
${ }^{1}$ http://falsifiable.us

${ }^{2}$ https://github.com/systemslab/popper

${ }^{3}$ https://en.wikipedia/wiki/DevOps
}

Permission to make digital or hard copies of part or all of this work for personal or classroom use is granted without fee provided that copies are not made or distributed for profit or commercial advantage and that copies bear this notice and the full citation on the first page. Copyrights for thirdparty components of this work must be honored. For all other uses, contact the owner/author(s).

PPoPP '19, February 16-20, 2019, Washington, DC, USA

(C) 2019 Copyright held by the owner/author(s).

ACM ISBN 978-1-4503-6225-2/19/02 . \$ \$15.00

https://doi.org/10.1145/3293883.3302575
Modern open source software development communities have created tools that make it easier to manage large codebases, allowing them to deal with high levels of complexity, not only in terms of managing code changes, but with the entire ecosystem that is needed in order to deliver changes to software in an agile, rapidly changing environment. These practices and tools are collectively referred to as DevOps. The Popper Experimentation Protocol repurposes the DevOps practice in the context of scientific explorations so that researchers can leverage existing tools and technologies to maintain and publish scientific analyses that are easy to reproduce.

In the first part of this short course, we will briefly introduce DevOps and give an overview of best practices. We will then show how these practices can be repurposed for carrying out scientific explorations and illustrate using some examples. The second part of the course will be devoted to hands-on experiences with the goal of walking the audience through the usage of the Popper CLI tool.

\section{Outline of tutorial}

- Introduction to the practical aspects of reproducibility.

- Overview of best practices in DevOps / open source software projects.

- Illustrate how Popper repurposes DevOps to scientific explorations.

- Show examples of projects that follow the Popper protocol.

- Hands-on experiences with the Popper CLI tool.

\section{Knowledge gap this course addresses}

Traditional experimentation practices are deeply rooted in the muscle memory of researchers: typing commands in "live" systems and getting results as they go. Popper puts an emphasis on versioning, automation and portability of experimentation pipelines. In practice, this means writing scripts (instead of directly typing on the terminal or GUIs), and making use of automation (DevOps) tools to execute these scripts. By following the Popper protocol, researchers can create the habit of generating scientific explorations that are easy to replicate, and amenable to collaboration/extension. 


\section{Prerequisites}

- Unix environment (Linux, MacOS or Linux Subsystem for Windows 10+).

- Bash $^{4}$, Git/Github ${ }^{5}$, Python, virtualenv ${ }^{6}$ and Docker ${ }^{7}$. Familiarity with these tools will make it easier to follow the Popper lesson ${ }^{8}$. In the case of Docker, familiarity with how container images are created ${ }^{9}$ is desired (but not strictly required).

- Popper CLI tool. Instructions on how to install can be found here ${ }^{10}$. Quickstart guide can be found here ${ }^{11}$.

- We can use an experimentation pipeline in your domain as the basis of our last hands-on exercise. This can be any analysis pipeline, parameter sweep for a simulation, or any other computational or data science study you would like to "popperize".

\section{Acknowledgements}

This work was partially funded by the Center for Research in Open Source Software ${ }^{12}$, Sandia National Laboratories and NSF Award \#1450488.

\section{References}

[1] I. Jimenez, M. Sevilla, N. Watkins, C. Maltzahn, J. Lofstead, K. Mohror, A. Arpaci-Dusseau, and R. Arpaci-Dusseau, "The Popper Convention: Making Reproducible Systems Evaluation Practical," 2017 IEEE International Parallel and Distributed Processing Symposium Workshops (IPDPSW), 2017, pp. 1561-1570.

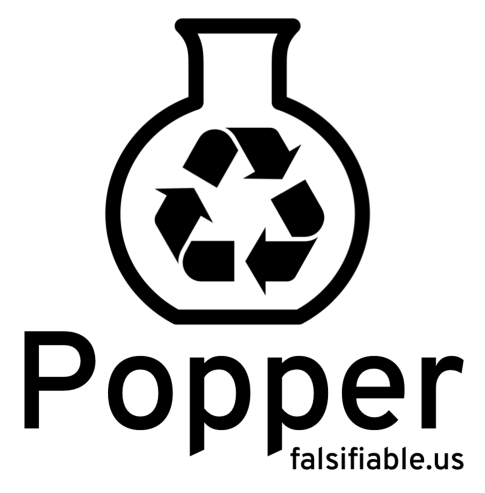

\footnotetext{
${ }^{4}$ http://swcarpentry.github.io/shell-novice/

${ }^{5}$ http://swcarpentry.github.io/git-novice/

${ }^{6} \mathrm{https}: / /$ realpython.com/python-virtual-environments-a-primer

${ }^{7}$ https://docker-curriculum.com/

${ }^{8}$ https://popperized.github.io/swc-lesson/

${ }^{9}$ https://docs.docker.com/get-started/part2/

${ }^{10}$ https://github.com/systemslab/popper/tree/master/cli\#install

${ }^{11}$ https://popper.readthedocs.io/en/latest/sections/getting_started.html

${ }^{12}$ https://cross.ucsc.edu
} 